\title{
Investigation and Analysis of a Cluster, of Influenza-like Cases in an Ethnic High School in Jingxi City, Guangxi
}

\author{
Wang Fuchun \\ Jingxi City Center for Disease Control and Prevention, Jingxi, China \\ Email address: \\ jxcdc2376@163.com
}

To cite this article:

Wang Fuchun. Investigation and Analysis of a Cluster, of Influenza-like Cases in an Ethnic High School in Jingxi City, Guangxi. International Journal of Infectious Diseases and Therapy. Vol. 6, No. 2, 2021, pp. 45-49. doi: 10.11648/j.ijidt.20210602.11

Received: March 11, 2021; Accepted: March 26, 2021; Published: April 1, 2021

\begin{abstract}
Objective: Analyze the epidemiological characteristics of a cluster of influenza-like cases in a national high school in Jingxi City, Guangxi, to provide a basis for formulating influenza prevention and control strategies and measures. Methods: Descriptive epidemiological methods are used to describe and analyze the epidemiological characteristics of influenza-like cases. Results: A total of 48 student influenza-like cases were reported from April 8 to 21,2020, with an attack rate of 4.46\% (48/1076), 20 males and 28 females, with a male-to-female ratio of 1:1.4; the main clinical feature is fever Accompanied by sore throat, cough, dry throat, sore throat, tonsil enlargement and other respiratory symptoms. The patient's boby temperature was as high as $38.9^{\circ} \mathrm{C}$, There were 15 people at $38.6-38.9^{\circ} \mathrm{C}$, accounting for $31.25 \%$, and 33 people at $38.0-38.5^{\circ} \mathrm{C}$, accounting for $67.75 \%$. The time of onset was April 14 with the largest number of patients, accounting for $18.75 \%$. The incidence class was based on 2017 (15), The number of patients in the class was the largest, accounting for $16.67 \%$. The age of onset was the largest in the 18-year-old age group, accounting for $72.92 \%$; three batches of throat swab specimens from some patients were collected, a total of 29 people were sent to the Guangxi Baise City Center for Disease Control and prevention for experiment Laboratory tests showed that all samples in the three batches were negative for the nucleic acid test of the new coronavirus pneumonia virus and the influenza virus. Conclusion: According to the epidemiological characteristics, combined with the patient's clinical manifestations and laboratory test results, the epidemic was determined to be a cluster of influenza-like cases. After timely prevention and control measures were taken, the epidemic was effectively controlled.
\end{abstract}

Keywords: Influenza-like Cases, Cluster Epidemic Situation, Investigation and Analysis

\section{Introduction}

From April 8 to 21, 2020, a cluster of influenza-like cases occurred in Jingxi Minority High school in Guangxi. After receiving the school epidemic report, we immediately organized professional and technical personnel to carry out on-site epidemiological investigations, and collected throat swab specimens for experiment Laboratory testing, preventive and control measures are taken to deal with the epidemic, the results are now reported as follows.

\section{Background}

Guangxi Jingxi City is Located on the China-Vietnam border in southwestern Guangxi, mbordering Vietham to the south, with a border line of $152.5 \mathrm{~km}$, an average elevation of
$750 \mathrm{~m}$, and an average annual temperature of $19.1^{\circ} \mathrm{C}$, It has 19 towns and villages with a total population of 670,000 . The ports that communicate with Vietnam include Longbang National First-Class Port and Yuexu National Second-Class Port. It is the most populous county-level city in the border counties of Guangxi and Vietnam. It is a county-level tourist city in Guangxi, with frequent personnel flow and active border trade. The city is an economically underdeveloped area with low economic and cultural living standards, poor awareness of the prevention of respiratory infectious diseases such as influenza, and influenza, epidemics in crowded places such as primary and secondary schools.

\section{Literature Review}

Influenza is a respiratory infectious disease monitored 
globally. Due to its rapid spread, influenza viruses are extremely prone to mutate, so influenza epidemics of different scales occur every year. Since the twentieth century, there have been 5 influenza pandemics in the world. The first 1918-1919 "Spanish influenza" Pandemic was caused by the H1N1 Subtype influenza virus, causing more than 700 million people worldwide to be infected, with an estimated 2500 Ten thousand to 40 million died. The second 1957 "Asian Influenza" pandemic was caused by the H2N2 subtype influenza virus and caused approximately 1 million deaths worldwide. The third "Hong kong flu" pandemic in 1968 was caused by the H3N2 subtype influenza virus, which caused 800,000 to 1 million deaths worldwide. The fourth "Russian flu" Pandemic in 1977 was caused by the H1N1 subtype influenza virus. The fifth 2009 H1N1 influenza pandemic was caused by a new H1N1 subtype influenza virus, causing 500,000 infections and 18,000 deaths worldwide, The H1N1 influenza pandemic in China is relatively serious, as of 2010 on May 30, a tota of 127839 confirmed cases were reported in 2340 counties and districts in 31 provinces, including 8331 severe cases, 805 deaths, and 2329 clusters of influenza A (H1N1); Guangxi is the same as the whole country. After the end of the influenza A H1N1 Pandemic in 2009, the influenza A H1N1 epidemic was seasonal; in Jingxi City, Local epidemics of influenza A H1N1, A H3N2 and B influenza occurred in many places, and Primary and Secondary Schools were the local influenza areas. In the main sites of outbreaks, monitoring of influenza epidemics should be strengthened, influenza Prevention and control knowledge Publicity and education should be carried out, personal protection should be strengthened, and the annual influenza vaccination rate should reach $80 \%$ or more, and the immune level of susceptible people should be improved. This is an effective way to prevent influenza clustered epidemics. Fundamental measures.

\section{Materials and Methods}

\subsection{Data Sources}

The epidemic data comes from the Jingxi City Influenza-like cases Network Reporting Information System in April 2020, on-site epidemiological investigations and patient case investigation registrations and other epidemic monitoring data. The population data of the school's students, faculty and staff are from Jingxi City provided by the Education Bureau.

\subsection{Case Diagnosis}

\subsubsection{Case Definition}

The case diagnosis criteria are strictly in accordance with the "Guidelines for the Handling of Influenza-like Case outbreaks (2018 Edition)"issued by the Bureau of Disease control and prevention of the National Health commission of China. Influenza-like cases are defined as clinical manifestations with fever and body temperature $\geq 38^{\circ} \mathrm{C}$, accompanied by cough or sore throat. patients who lack other laboratory confirmation can be diagnosed as influenza-like cases [1].

\subsubsection{Epidemiological Investigation}

Investigation and search for all patients whose teachers, students and staff of the school became ill during the epidemic, and uniformly trained epidemiological investigation professionals will use a unified influenza-like case investigation form to investigate and register one by one case, Verification of the diagnosis, all patients are treated in isolation on the spot, home isolation or centralized isolation medical observation after the condition improves, the body temperature. returns to normal, and the isolation is lifted 48 after the symptoms disappear, Investigate the implementation of the school's morning and afternoon, body temperature testing and the registration and inspection system for teachers and students who are absent from school, monitor the school's epidemic report and influenza vaccination in recent years, inspect and guide the school's environmental sanitation, indoor ventilation and disinfection, and health and epidemic prevention, and put forward suggestions for epidemic prevention and control measures.

\subsubsection{Laboratory Testing}

Collect throat swab specimens of some patients on site, place them in specimen collection tubes. containing sterile virus sampling solution, and store them at $4^{\circ} \mathrm{C}$ on ice. Within 48 hours, it will be sent to the laboratory of the Guangxi Baise City center for Disease Control and prevention, and the new coronavirus nucleic acid test and influenza virus nucleic acid test will be carried out by the fluorescence quantification method (RT-PCR).

\subsection{Methods}

Using descriptive epidemiological methods. the data of influenza-like cases were statistically analyzed by Excel 2003 software, $\mathrm{x}^{2}$ test was used for counting data, and $\mathrm{P}<0.05$ was considered statistically significant.

\section{Results}

\subsection{Basic Information}

Jingxi Minority High school was founded in 2015, It is a full-time public high school. The school is located next to Deai Avenue, chengbei, Jingxi City. The campus covers an area of 94, 000 square meters and a building area of 62, 500 square meters. There are 3, 216 students in 60 classes in grades 1 to 3 of high school, 279 faculty members, 68 full-time teachers, and 3 school doctors. Affected by the new crown pneumonia epidemic, the school has 20 classes in the third grade of high school with 1076 students and 62 faculty members. The first batch of classes will resume on April 7 th. The remaining class students and faculty will resume classes for the second batch on April 22. A cluster of influenza-like cases occurred in the school from April 8 to 21, 2020. A total of 48 cases were reported and no deaths occurred. After receiving the epidemic report, the Jingxi City Center for 
Disease Control and prevention organized presonnel to promptly rush to the scene for investigation and disposal.

\subsection{Epidemiological Characteristics}

\subsection{First Case}

The school's 2017 (13) grade 3 student Zhou Moumou, female, 19 Years old, began to feel unwell in the afternoon of April 8. The clinical manifestations were mainly fever and body temperature of $38^{\circ} \mathrm{C}$, accompanied by cough, sore throat and other symptoms. He went to Jingxi City Hospital of Traditional Chinese Medicine and was clinically diagnosed as an upper respiratory tract infection. He was then given symptomatic treatment for improvement, and was taken home isolation medical observation. On April 17, his body temperature returned to normal, his symptoms disappeared and the returned to school for class. The patient's family lived in the urban area of Jingxi City and had no history of going out before the illness. None of the family members and surrounding neighbors had similar symptoms.

\subsubsection{Time Distribution}

The school reported a total of 48 influenza-like cases from April 8 to 21,2020. The patients were all students. The faculty and staff had no case reports. The onset time was mainly from April 11 to 17.39 cases occurred, accounting for $81.25 \%$, as shown in Figure 1.

\subsubsection{Class Distribution}

Among the 20 classes in the thrid grade of high school, 15 classes have reported cases. The 2017 (15) class has a higher incidence of $16.67 \%(8 / 48)$, and the rest of the class has fewer cases, the difference in class incidence is statistically significant $\left(\mathrm{x}^{2}=41.14, \mathrm{P}<0.01\right)$ as shown in Table 1 .

\subsubsection{Clinical Characteristics}

The clinical manifestations of patients are mainly fever, accompanied by cough, sore throat, and a few are dry throat, dizziness, sore throat, and tonsil enlargement. The 48 cases were accompanied by their parents to the hospital for diagnosis and treatment. The clinical diagnosis of the above-mentioned respiratory tract infection is mainly the incubation period of 1 to 5 days, of which 42 cases have an incubation period off 2 to 3 days, accounting for $87.50 \%$, and 33 cases have a fever (axillary temperature) of $38.0-38.5^{\circ} \mathrm{C}$. Accounted for $68.75 \%, 15$ cases were $38.6-38.9^{\circ} \mathrm{C}$, accounting for $31.25 \%$, See Table 2 .

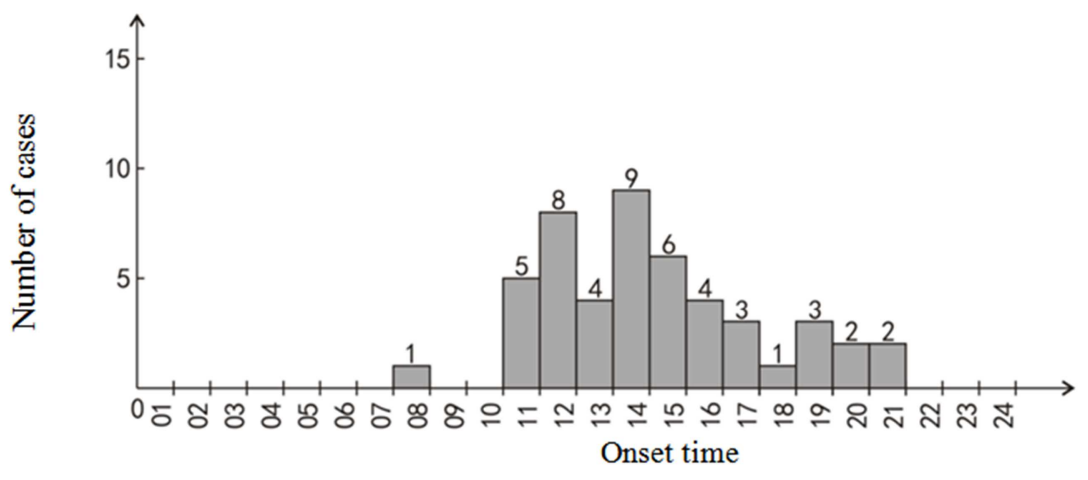

Figure 1. Time distribution of influenza-like cases in ethnic high school in Jingxi City in April 2020.

Table 1. Class distribution of influenza-like cases in Jingxi City Ethnic High School in April 2020.

\begin{tabular}{lllll}
\hline Class & Student number & Number of cases & Attack rate (\%) & Composition ratio (\%) \\
\hline 2017 (1) Calss & 49 & 0 & 0.00 & 0.00 \\
2017 (2) Calss & 49 & 1 & 2.04 & 2.08 \\
2017 (3) Calss & 49 & 1 & 2.04 & 2.08 \\
2017 (4) Calss & 51 & 0 & 0.00 & 0.00 \\
2017 (5) Calss & 58 & 2 & 3.45 & 4.17 \\
2017 (6) Calss & 56 & 2 & 3.57 & 2.08 \\
2017 (7) Calss & 51 & 1 & 1.96 & 0.00 \\
2017 (8) Calss & 58 & 0 & 0.00 & 10.42 \\
2017 (9) Calss & 56 & 5 & 8.93 & 0.00 \\
2017 (10) Calss & 53 & 0 & 0.00 & 10.42 \\
2017 (11) Calss & 50 & 5 & 10.00 & 8.33 \\
2017 (12) Calss & 52 & 4 & 7.69 & 2.08 \\
2017 (13) Calss & 49 & 1 & 2.04 & 2.08 \\
2017 (14) Calss & 47 & 1 & 2.13 & 16.67 \\
2017 (15) Calss & 58 & 8 & 13.79 & 6.25 \\
2017 (16) Calss & 58 & 3 & 5.17 & 10.42 \\
2017 (17) Calss & 57 & 5 & 8.77 & 0.00 \\
2017 (18) Calss & 58 & 0 & 0.00 & 8.33 \\
2017 (19) Calss & 57 & 4 & 7.02 & 10.42 \\
2017 (20) Calss & 60 & 5 & 8.33 & 100.00 \\
Total & 1076 & 48 & 4.46 & \\
\hline
\end{tabular}


Table 2. Clinical characterics of influenza-like cases in ethnic high school in Jingxi City in April 2020.

\begin{tabular}{lll}
\hline Symptom & Student number & Composition ratio (\%) \\
\hline Fever (armpit temperature) $38.0-38.5^{\circ} \mathrm{C}$ & 33 & 68.75 \\
Fever (armpit temperature) $38.6 \sim 38.9^{\circ} \mathrm{C}$ & 15 & 31.25 \\
Cough & 18 & 37.50 \\
Sore throat & 15 & 31.25 \\
Enlarged tonsils & 6 & 12.50 \\
Dizziness and headache & 5 & 10.42 \\
Muscle ache & 3 & 6.25 \\
Chills & 2 & 4.17 \\
\hline
\end{tabular}

\subsubsection{Population Distribution}

The 48 patients of this epidemic are the first batch of third-year high school students who started school and resumed classes. There are 20 males and 28 females. The male to female ratio is $1: 1.4$, The male attack rate was $3.78 \%$ (20/529), and the female attack rate was 5.12\% (28/547), the difference was not statistically significant $\left(\mathrm{x}^{2}=1.13, \mathrm{P}>0.05\right)$, and age of onset is 17-20 years old, The17-year-old group had 2 cases, accounting for $4.17 \%$, the 18 -year-old group had 35 cases, accounting for $72.92 \%$, the 19-year-old group had 10 cases. Accounting for $20.08 \%$, and the 20 -year-old group had 1 cases, accounting for $2.08 \%$. The attack rate of the $17-18$-year-old age group was $8.45 \%(37 / 438)$, and the 19-20-year-old age group had an attack rate of $1.72 \%$ $(11 / 638)$, Comparing the two, the17-18-year-old age group had an attck rate higher than 19-20-year-old age group $\left(\mathrm{x}^{2}=27.54, \mathrm{P}<0.01\right)$. After investigation, all cases had no history of influenza vaccination in the past year.

\subsection{Laboratory Testing}

According to the on-site investigation, throat swab specimens from some patients were collected in three batches on April 12, 13, and 16 and sent to the laboratory for testing in 6, 8, and 15 for a total of 29 According to the laboratory of Baise City Center for Disease Control and prevention, pharyngeal swab samples were tested for new coronary pneumonia virus nucleic acid and influenza virus nucleic acid by fluorescence quantitative method (RT-PCR). The results showed that 29 people from three batches of specimens were all new coronary pneumonia. Viral nucleic acid test and influenza virus nucleic acid test were negative.

\section{Discussion}

Influenza (influenza, abbreviated as influenza) is an acute respiratory infectious disease caused by influenza virus. It is highly contagious, spreads quickly, and has serious harm. Influenza virus has the characteristics of antigenic variability and general susceptibility to the virus [2-4], has caused many pandemics worldwide, causing millions of people to get sick and up to 1 million deaths worldwide every year. It is the first infectious disease to be monitored globally [5]. Influenza viruses are classified into influenza $\mathrm{A}, \mathrm{B}$ and $\mathrm{C}$ according to the antigenicity of nucleoprotein (NP) and matrix protein (M1) [6]. Influenza A and B are easily Influenza outbreaks are caused, especially the influenza A H3N2 virus has a higher prevalence, followed by the A H1N1 influenza virus and B influenza virus [7]. People infected with a certain subtype of influenza virus will produce antibodies to that type of influenza virus, So influenza virus subtypes generally appear alternately. Influenza vaccination is currently the best way to prevent and control influenza [8].

The results of the on-site epidemiological investigation indicate that the occurrence of this epidemic may be related to the weak links in the prevention and control of the epidemic in the school: (1) The school is not very aware of the prevention of respiratory infectious disease. In the early stage of the epidemic, students in the class with the onset of the disease did not restrict activities between classes, resulting in the source of infection not being well controlled. (2) The epidemic report is not timely enouhg. After the first patient, the epidemic was not reported immediately. It was not until the 5th day of the epidemic when the number of students increased, which affected normal schooling, and school did not report the epidemic, which affected the timely impalementation epidemic investigation and disposal and prevention and control measures. (3) The sensitivity of clinical diagnosis of influenza-like cases by clinicians and school doctors in medical institutions is not high. At the beginning of the onset, the doctors were often misdiagnosed as upper respiratory tract infection or common cold, and the patients and their families were not reminded in time to do isolation treatment and personal protection. (4) school hygiene and disease prevention puplicity is not adequate. school teachers and students lacked knowledge about the prevention and control of influenza-like cases. All patients in this epidemic had not received influenza vaccine in the past year. (5) Insufficient indoor ventilation in cold weather. At the beginning of the school's resumption of classes, the temperature is colder and the temperature difference between morning and evening is relatively large. It is not enough to maintain air circulation in classrooms, dormitories, canteens and other places. Students are crowded in the same room, and close contact increases the chance of infection.

Schools are densely populated and are places with a high incidence of respiratory infectious disease [9-10]. According to surveys, more than $85 \%$ of influenza outbreaks occur in primary and secondary schools [11]. Therefore, timely reporting of the epidemic and investigation and disposal play a key role in controlling the epidemic. Primary and secondary schools should conscientiously implement patient isolation and treatment, Publicity and education, disinfection and ventilation, strengthen morning and afternoon inspections and 
epidemic surveillance reports, strengthen personal protection, wear masks, wash hands frequently, refrain from gathering, develop good personal hygiene habits and other comprehensive measures to control infection sources, cutting off the route of transmission, actively carrying out influenza vaccination work, and increasing the influenza vaccination rate of susceptible people [12-14], Are the fundamental measures to effectively control the influenza epidemic.

\section{Conclusion}

According to epidemiological investigations, combined with clinical manifestations and laboratory test results, in accordance with the definition and diagnostic criteria of influenza-like cases [15], it is confirmed that this epidemic is a cluster of influenza-like cases. The corresponding preventive and control measures include: (1) Strengthen epidemic surveillance reports, and isolate and treat patients in a timely manner. (2) Follow up and register the teachers and students who are absent due to illness. (3) Strengthen indoor ventilation and disinfection, maintain air criculation and disinfection in classrooms, dormitories, canteens and other places, and keep the environment clean and hygienic. (4) Strengthen the publicity and education of hygiene and disease prevention, Combined with the Prevention and control of the new crown pneumonia epidemic, take personal protection, wear masks, wash hands frequently, do not gather, and seek medical treatment in time if symptoms such as fever occur. After timely measures to deal with the epidemic, the epidemic was quickly and affectively controlled.

\section{References}

[1] The Disease Control and Prevention Bureau of the National Health Commission of the People's Republic of China, Guidelines for Handling Outbreaks of influenza-like Cases (2018 Edition), EB/OL-November 14, 2018.

[2] Yu Xuelan, Zeng Jiarui, Xu Yong, et al (2019). Analysis of Influenza Surveillance Results from 2016 to 2017 in Neijiang City, Sichuan Province, Medical Animal Control, 35 (11): 1063-1065.

[3] Lu Xufeng, Zhang Huili, Han Changlei (2019). Investigation on an outbreak of influenza A HINI in primary school in changzhou City, Jiangsu province, Medical Animal Control, 35 (11): 1109-1111.

[4] Zhang Guobao, Chen Fengyang, Dong Jianghua, et al (2018). Analysis of Etiological Surveillance Results of influenza in Xingtai City, Hebei Province from 2014 to 2016, Medical Animal Control, 34 (6): 547-549.

[5] Liu Zhenrong, Li Lanyan, Liu Dan, et al (2017). 2011-2015 Epidemic Characteristics of Influenza and Research on Prevention and Control Strategies in Tangshan City, Hebei Province, Medical Animal Control, 33 (9): 537-539.

[6] Li Lanjuan, Ren Hong, Infectious Disease, 8rd ed, Beijing: people's Medical Publishing House, 2015, pp. 193-196.

[7] Lui Yingxia, Li Shanshan, Liu Yao, et al (2016). Analysis of Etiological Test Results of Influenza-like cases in Dongcheng District, Beijing in 2014, International Journal of Virology, 23 (5) $321-331$.

[8] Wu Shuangzhi, Liu Yang, Liu Lei, et al (2016). Analysis of Influenza Surveillance Results in Mudanjiang City from 2013 to 2014, Medical Animal Control, 32 (5): 546-548.

[9] Peng Liying, Zhang Hua, Lei Jiezhen (2016). Monitoring and analysis of influenza etiology in Yunfu City from 2010 to 2014, Practical Preventive Medicine, 23 (11): 1374-1377.

[10] Zhou Lijun, Liu Xuecheng, Lu Qiang, et al (2016) Analysis of the results of influenza Surveillance in Sichuan Province from 2011 t0 2014, Occupational and Injuries, 31 (1): 24-27.

[11] An Zhou, Cheng Zhouxiang, Qian Bangqun, et al (2016). Epidemiological characteristics analysis of the outbreak of influenza-like cases in schools in Wuhu City from 2013 t0 2015, Modern Preventive Medicine, 43 (22): 4053-4056.

[12] Liu Haibo, Chen Dating, Li Xinxin, et al (2017). Analysis of influenza cluster epidemic and vaccine Protection rate in Daxing District, Beijing from 2014 to 2016, Modern preventive Medicine, 44 (19): 3539-3541.

[13] JEFFERSON T, RIVETTI A, DI PC, et al (2010). Waccines for preventing influenza in healthy children, Cochrane Database Syst Rev, 1 (2): 367-522.

[14] Wang Fuchun, Huang Zhending, Xu Shaoren, et al (2018). Analysis of the epidemiological characteristics of a school outbreak of H1N1 influenza, Preventive Meticine Forum, 24 (12): 919-921.

[15] Ministry of Health of the People's Republic of China, ws 285-2008 Influenza diagnostic criteria, Beijing: People's Medical Publishing House, 2008. 